\title{
Soft, entirely photoplastic probes for scanning force microscopy
}

\author{
G. Genolet, ${ }^{\text {a) }}$ J. Brugger, M. Despont, U. Drechsler, and P. Vettiger \\ IBM Research Division, Zurich Research Laboratory, CH-8803 Rüschlikon, Switzerland
}

N. F. de Rooij

Institute of Microtechnology, University of Neuchâtel, CH-2000 Neuchâtel, Switzerland

D. Anselmetti

Novartis Services AG, Scientific Services, Physics, K-127.620, CH-4002 Basel, Switzerland

(Received 29 October 1998; accepted for publication 10 February 1999)

\begin{abstract}
A new probe made entirely of plastic material has been developed for scanning probe microscopy. Using a polymer for the cantilever facilitates the realization of mechanical properties that are difficult to achieve with classical silicon technology. The new cantilever and tip presented here are made of an epoxy-based photoplastic. The fabrication process is a simple batch process in which the integrated tip and the lever are defined in one photolithography step. The simplicity of the fabrication step, the use of a polymer as material, and the ability to reuse the silicon mold lead to a soft low-cost probe for scanning force microscopy. Imaging soft condensed matter with photoplastic levers, which uses laser beam deflection sensing, exhibits a resolution that compares well with that of commercially available silicon cantilevers.
\end{abstract}

\section{INTRODUCTION}

Since its development in $1986,{ }^{1}$ the scanning force microscope (SFM) has become a revolutionary tool for surface analysis. In a SFM, a very sharp tip located at the end of a cantilever is scanned over the sample surface. The tipsample interaction is converted by the cantilever into a displacement measured by a deflection sensor. Optical interferometry, ${ }^{2,3}$ and capacitive, ${ }^{4}$ and piezoresistive ${ }^{5}$ methods can be used to sense the deflection of the lever, but the laser beam deflection technique ${ }^{6}$ is still the most widely used.

The construction of a force-sensing cantilever probe has remained a critical issue in SFMs. Micromachining technologies have allowed batch fabrication of SFM probes made of silicon, ${ }^{7}$ silicon nitride, or silicon oxide with integrated or add-on tips. ${ }^{8}$ For SFM applications, the cantilever must have certain mechanical properties. Low stiffness, a high resonant frequency, and a fairly high $Q$ factor together with a sharp tip are the main criteria that must be fulfilled by such a probe. The stiffness of a cantilever is given by its spring constant $k=E w t^{3} / 4 l^{3}$ where $w, t$, and $l$ denote the width, thickness, and length of the lever, and $E$ is the Young's modulus of the material used. When an optical beam is used to measure the deflection, the sensitivity of the detector is inversely proportional to the length of the cantilever. ${ }^{6}$ Unfortunately, short levers are a drawback if a low spring constant is required. A certain width is also necessary for sufficient reflection and simple alignment of the laser beam. In addition, silicon and silicon nitride have a high Young's modulus $E$ (190 and 385 $\mathrm{GPa}$, respectively ${ }^{9}$ ). With such materials, the only way to

${ }^{a)}$ Also at the Institute of Microtechnology, University of Neuchâtel, JaquetDroz 1, CH-2000 Neuchâtel, Switzerland; electronic mail: gen@zurich.ibm.com obtain a low spring constant $(<5 \mathrm{~N} / \mathrm{m})$ is to reduce the thickness of the cantilever, but very small thicknesses $(<1 \mu \mathrm{m})$ are technologically difficult to achieve. Alternatively, fabrication of soft cantilevers with noncritical dimensions is possible by taking a material with a low Young's modulus. Pechmann et al. ${ }^{10}$ reported the fabrication of very soft cantilevers using a novolak photoresist as material. The fabrication process is nevertheless complex, and the tip is placed on the lever by electron-bean deposition (EBD), a technique that is not well suited for high-volume fabrication.

We present a new, simple fabrication technique for soft, entirely photoplastic SFM probes with integrated tips made with a batch molding technique.

\section{DESCRIPTION AND FABRICATION OF PHOTOPLASTIC PROBES}

Invented and developed by IBM, ${ }^{11,12}$ the SU-8 photoplastic used to create the soft cantilevers is a negative-tone photoresist consisting of the epoxy-based EPON® SU-8

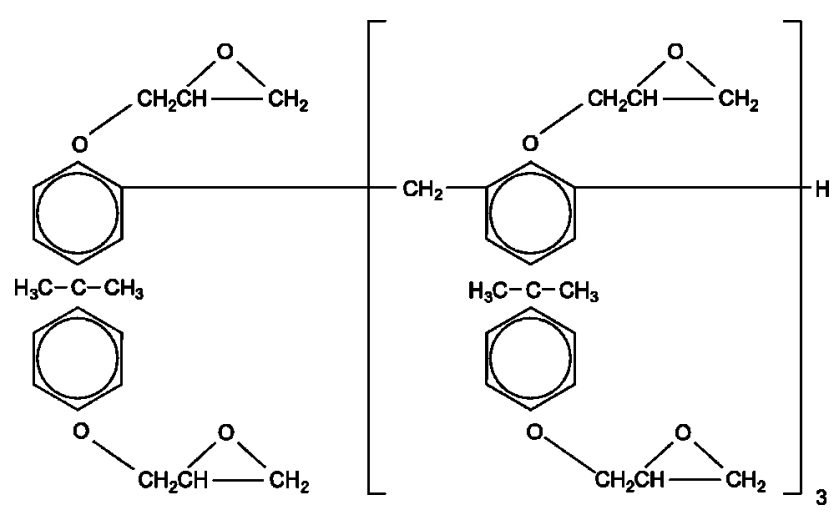

FIG. 1. Molecular structure of SU-8 with its eight epoxy sites. 


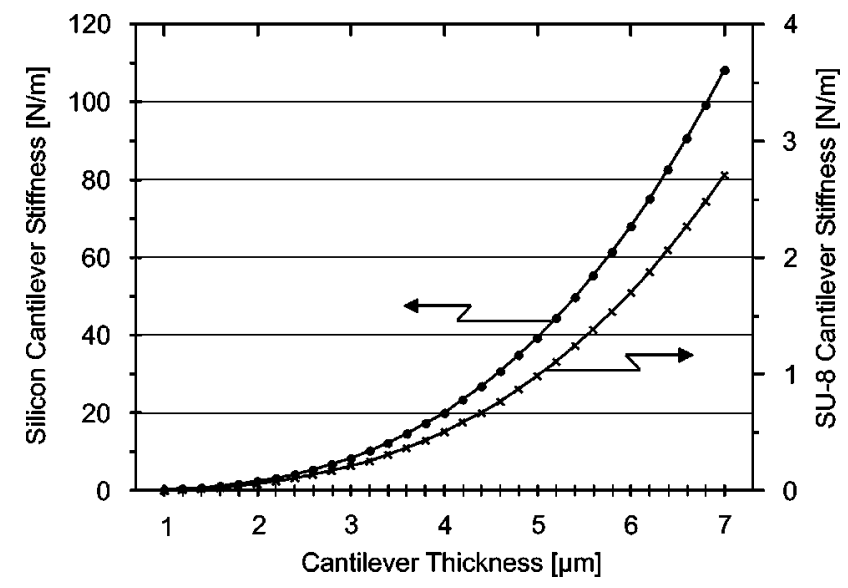

FIG. 2. Spring constant of silicon and SU-8 cantilevers as a function of thickness. Cantilevers have a length of $156 \mu \mathrm{m}$ and a width of $30 \mu \mathrm{m}$.

resin (by Shell Chemical) photosensitized with a triaryl sulfonium salt (CYRACURE® UVI by Union Carbide). This resist features low-cost lithography, galvanoforming, and abformung (LIGA) ${ }^{13}$ which produces a high aspect ratio without the need for an x-ray source. Such features have become especially important in the growing field of microelectromechanical systems (MEMS) applications. ${ }^{14,15}$

$\mathrm{EPON} ® \mathrm{SU}-8$ is a negative photoresist with the highest epoxide functionality per molecule commercially available (see Fig. 1). The highly crosslinked structure induced by ultraviolet exposure gives SU-8 its high thermal stability and good chemical resistance. In addition to these properties, an aspect ratio as high as 18 and a low Young's modulus ( $E$ $\cong 4 \mathrm{GPa}$ ) make this photoplastic a suitable material for micromechanical structures for which a low spring constant is required. ${ }^{16}$ Moreover, this material is very promising for the simple fabrication of all photoplastic SFM probes with integrated tips and a low spring constant. Figure 2 compares the spring constants of silicon and SU-8 cantilever beams of equal length and width as a function of thickness. It clearly shows that very low spring constants can easily be achieved with fairly thick SU-8 levers. In contrast, levers with the same spring constant made of silicon must be very thin, which is difficult to achieve and control.

The fabrication process of SU-8 photoplastic cantilevers is based on the spin coating and exposure/development of the

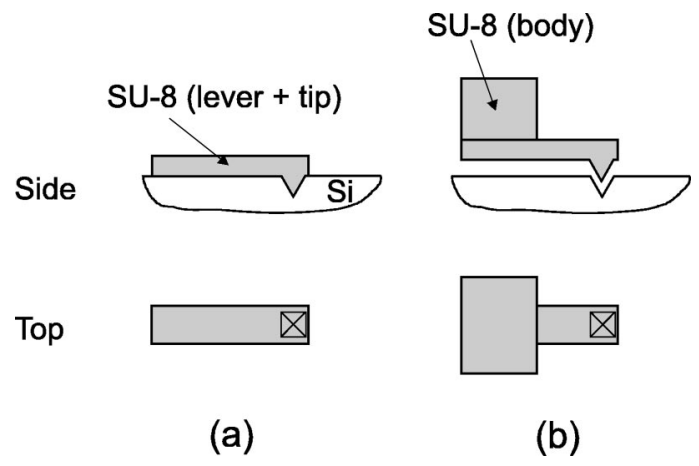

FIG. 3. (a) Formation of an SU-8 cantilever. The tip is formed by the resist molded in the pyramidal pit. (b) Free-standing cantilever is obtained upon unmolding of the chip.
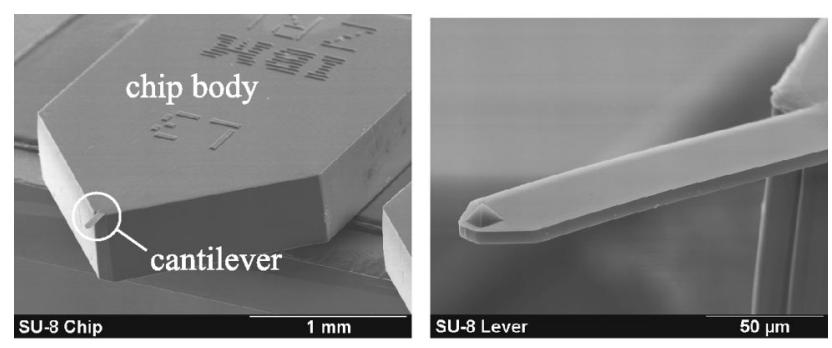

(a)

(b)

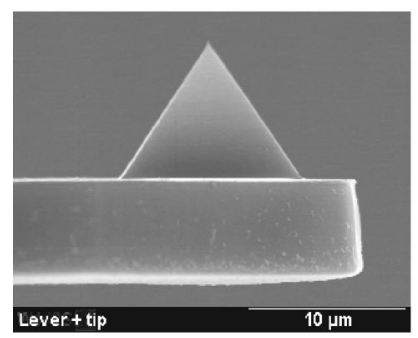

(c)

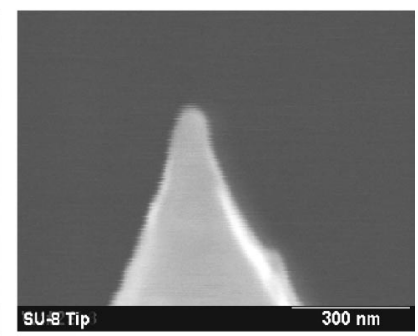

(d)

FIG. 4. (a) General view of an SU-8 SFM probe. (b) Close up of an SU-8 lever. (c) Close-up of a pyramidal tip at the end of the lever. (d) Close-up of an SU-8 tip. Radius of curvature is approximately $30 \mathrm{~nm}$.

SU-8 photoresist on a silicon wafer. These steps are repeated for subsequent layers. Cantilevers with integrated tips are fabricated in a very similar manner as that in which Albrecht et al. made silicon nitride cantilevers. ${ }^{8}$

The initial material is a $100 \mathrm{~mm}\langle 100\rangle$ silicon wafer with $1 \mu \mathrm{m}$ of thermally grown oxide on both sides. The topside is patterned using $\mathrm{CHF}_{3} / \mathrm{O}_{2}$ reactive ion etching (RIE) to form the mask for etching the tip molds. Pyramidal holes are then etched in the silicon wafer using an anisotropic etchant such as $\mathrm{KOH}$. In order to improve the sharpness of the SU-8 tips, the molds are sharpened in a thermal oxidation step. ${ }^{17}$ Tip sharpness depends mainly on the quality of the mold.

A first layer of SU-8 is then spin coated onto the wafer, and the tip is defined by the resist, which fills up the etched pyramidal sharp holes. The latter act as molds. The cantilever's thickness is determined by the thickness of the first resist layer as shown in Fig. 3(a). Subsequently, the resist is exposed to contact printing with a $400 \mathrm{~nm}$ ultraviolet source to form a crosslinked volume, which will not be dissolved in the subsequent development step, and hence, defines the dimensions of the cantilever. A second layer of photoresist is then spin coated, exposed, and developed to form the body of the chip. Afterwards, levers are released using a sacrificial

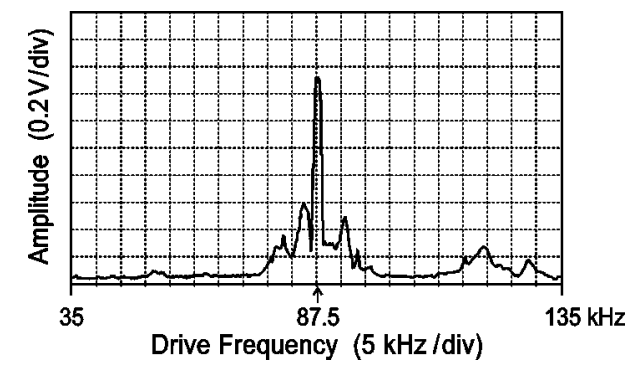

FIG. 5. Frequency response of an SU-8 cantilever. Dimensions are $t=6.6$ $\mu \mathrm{m}, w=31 \mu \mathrm{m}, l=150 \mu \mathrm{m}$. 


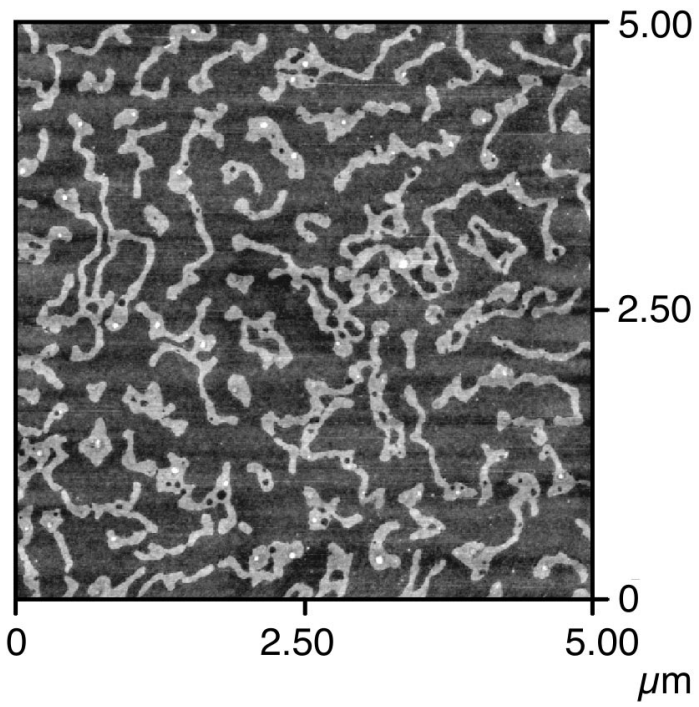

FIG. 6. AFM image $\left(5 \times 5 \mu \mathrm{m}^{2}\right)$ of a mixed Langmuir-Blodgett film with coexisting hydrocarbon and fluorocarbon molecular domains. The image $z$ range corresponds to $5 \mathrm{~nm}$ (see the text for details).

layer technique, and chips are removed mechanically from the silicon wafer mold without being damaged [see Fig. 3(b)]. Hence, after cleaning, the mold is ready to be reused for the fabrication of new chips. This is very important to minimize the cost of these plastic probes. Finally, $40 \mathrm{~nm}$ of gold was evaporated on the backside of the levers to achieve sufficient reflection for optical detection.

\section{EXPERIMENTS AND DISCUSSION}

Figure 4 shows scanning electron micrographs of fabricated photoplastic SU-8 SFM probes with integrated sharp tips. Thickness, width, and length of the levers are 6.6, 31, and $150 \mu \mathrm{m}$, respectively, which yield a calculated spring constant of $2.64 \mathrm{~N} / \mathrm{m}$ and a resonant frequency of $87 \mathrm{kHz}$. Measured resonant frequencies differ up to $5 \%$ from theoretical values. The difference is due mainly to the measurement accuracy of the lever dimensions and the approximations of

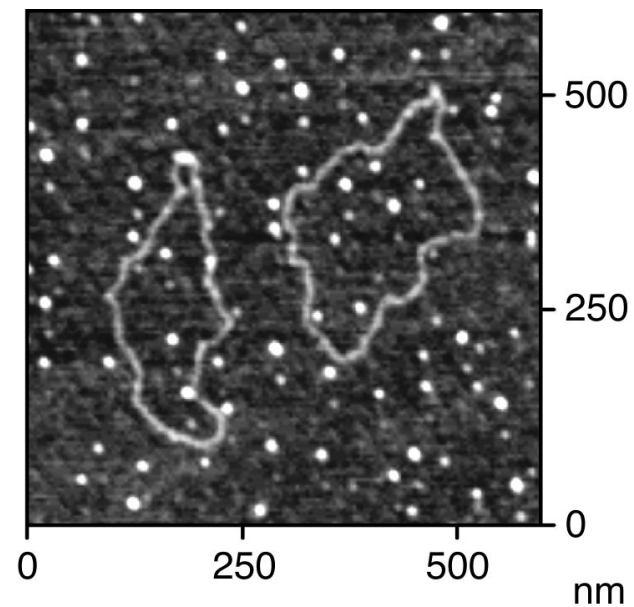

FIG. 7. AFM image $\left(0.6 \times 0.6 \mu \mathrm{m}^{2}\right)$ of DNA-plasmid (pGEM-3Zf vector from Promega Corporation, Madison, WI). The width of the DNA strand is $5-6 \mathrm{~nm}$. The $z$ scale was color adjusted to $1.5 \mathrm{~nm}$.

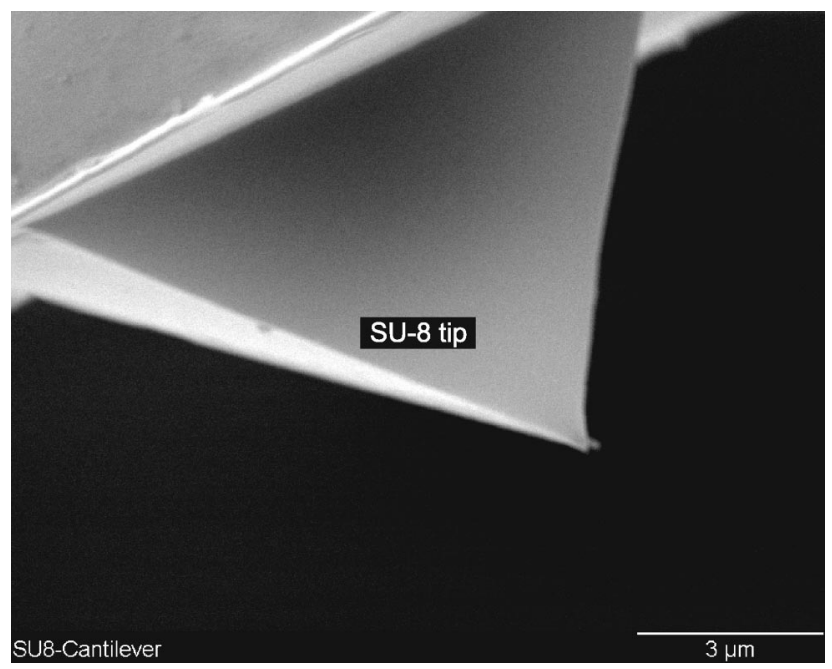

FIG. 8. SEM image of a tip after AFM imaging of soft samples. The tip showed no wear after imaging.

the density and Young's modulus of the SU-8 material. A $Q$ factor in air of about 50 was deduced from the frequency response in Fig. 5.

Tips produced in this manner have been measured to have radii of curvature of less than $30 \mathrm{~nm}$. Such values have been determined by scanning electron microscopy (SEM) measurements after $20 \mathrm{~nm}$ of gold had been sputtered on the tip. Hence, the actual radii of curvature of the photoplastic tips are somewhat smaller than indicated by SEM measurements.

The control of the cantilever thickness is a major challenge because the spring constant is proportional to the thickness cubed. The thickness of the photoplastic cantilevers described here is determined by the SU-8 spin-coating process. Uniformity and reproducibility of photoresist spin-coating processes are known to be very good, hence, cantilever stiffness and resonant frequency of photoplastic levers are well defined and controlled. For a target cantilever thickness of 6 $\mu \mathrm{m}$, a mean value of $5.91 \mu \mathrm{m}$ with a standard deviation of $0.076 \mu \mathrm{m}$ has been measured over a 4 in. wafer, resulting in a standard deviation of the spring constant of $0.073 \mathrm{~N} / \mathrm{m}$ assuming nominal length and width.

The performance of the epoxy cantilevers has been investigated in atomic force microscopy (AFM) experiments on so-called soft condensed-matter samples. The handling and resolution of the levers have been tested in the tapping mode under ambient conditions on mixed LangmuirBlodgett films (Fig. 6), DNA-plasmid molecules (Fig. 7), and M13 bacteriophage samples. Figure 6 clearly shows the coexisting phases (domains) of molecules of different hydrophobicity (surface energy). The lateral resolution can be estimated from Fig. 7, where the width of the DNA strand is 5-6 $\mathrm{nm}$. The vertical resolution was found to be of the order of $0.1 \mathrm{~nm}$. The background of Fig. 7 reveals contamination due to aging of the sample. Figure 8 illustrates that the tip showed no wear after images such as those in Figs. 6 and 7 were taken.

In summary, the fabrication of new, soft, photoplastic SFM probes with integrated tips has been demonstrated. The 
simple molding and replication techniques show interesting potential for the fabrication of low-cost, large-volume photoplastic SFM probes. The spin-coating process of thin layers $(<1 \mu \mathrm{m})$ will even make the fabrication of very soft cantilevers possible. In addition, tip hardening by overcoating will reduce or eliminate wear and may lead to very broad applications for such probes.

\section{ACKNOWLEDGMENTS}

The authors thank G. Binnig, U. Dürig, W. Häberle, M. Lutwyche, H. Rothuizen, and R. Widmer of the Micro- and Nanomechanics group at the IBM Zurich Research Laboratory for valuable discussions and help, M. Fujihira of the Tokyo Institute of Technology and F. Schwesinger of the Swiss Federal Institute of Technology, Zurich, for providing some of the biomaterials, and H. Lorenz of the EPFL for helpful advice on using SU-8.

${ }^{1}$ G. Binnig, C. F. Quate, and Ch. Gerber, Phys. Rev. Lett. 56, 930 (1986).

${ }^{2}$ D. Rugar, H. J. Mamin, R. Erlandson, J. E. Stern, and B. D. Terris, Rev. Sci. Instrum. 59, 2337 (1988).

${ }^{3}$ Y. Martin, C. C. Williams, and H. K. Wickramasinghe, J. Appl. Phys. 61, 4723 (1987).
${ }^{4}$ T. Göddenhenrich, H. Lemke, U. Hartmann, and C. Heiden, J. Vac. Sci. Technol. A 8, 383 (1990).

${ }^{5}$ M. Tortonese, R. C. Barrett, and C. F. Quate, Appl. Phys. Lett. 62, 834 (1993).

${ }^{6}$ N. M. Amer and G. Meyer, Bull. Am. Phys. Soc. 33, 319 (1988); G. Meyer and N. M. Amer, Appl. Phys. Lett. 53, 2400 (1988).

${ }^{7}$ O. Wolter, Th. Bayer, and J. Greschner, J. Vac. Sci. Technol. B 9, 1353 (1991).

${ }^{8}$ T. R. Albrecht, S. Akamine, T. E. Carver, and C. F. Quate, J. Vac. Sci. Technol. A 8, 3386 (1990).

${ }^{9}$ K. Petersen, Proc. IEEE 70, 420 (1982).

${ }^{10}$ R. Pechmann, J. M. Köhler, W. Fritzsche, A. Schaper, and T. M. Jovin, Rev. Sci. Instrum. 65, 3702 (1994).

${ }^{11}$ N. LaBianca and J. D. Gelorme, Proc. SPIE 2438, 846 (1994).

${ }^{12}$ K. Y. Lee, N. LaBianca, S. A. Rishton, S. Zolgharnain, J. D. Gelorme, J. Shaw, and T. H.-P. Chang, J. Vac. Sci. Technol. B 13, 3012 (1995).

${ }^{13}$ E. W. Becker, W. Ehrfeld, P. Hagmann, A. Maner, and D. Münchmeyer, Microelectron. Eng. 4, 35 (1985).

${ }^{14}$ M. Despont, H. Lorenz, N. Fahrni, J. Brugger, P. Renaud, and P. Vittiger, in Proceedings of the Tenth Annual International Workshop on Micro Electro Mechanical Systems. An Investigation of Micro Structures, Sensors, Actuators, Machines, and Robots (IEEE, Piscataway, NJ, 1997), pp. $518-522$.

${ }^{15}$ H. Lorenz, M. Despont, N. Fahrni, J. Brugger, P. Renaud, and P. Vettiger, Sens. Actuators A 64, 33 (1998).

${ }^{16}$ H. Lorenz, M. Laudon, and P. Renaud, Microelectron. Eng. 41/42, 371 (1998).

${ }^{17}$ S. Akamine and C. F. Quate, J. Vac. Sci. Technol. B 10, 2307 (1992). 